\title{
Ag@Au Concave Cuboctahedra: A Unique Probe for Monitoring Au-Catalyzed Reduction and Oxidation Reactions by Surface- Enhanced Raman Spectroscopy
}

Jiawei Zhang, ${ }^{\dagger \neq}$ Sarah A. Winget, ${ }^{\dagger}{ }^{\ddagger}$ Yiren $\mathrm{Wu}^{\dagger}{ }^{\dagger}$ Dong Su, ${ }^{\&}$ Xiaojun Sun, ${ }^{\dagger}$ Zhao-Xiong Xie, ${ }^{\ddagger}$ and Dong Qin ${ }^{\dagger, *}$

${ }^{\dagger}$ School of Materials Science and Engineering, Georgia Institute of Technology, Atlanta, Georgia 30332, United States

‡State Key Laboratory of Physical Chemistry of Solid Surfaces and Department of Chemistry, Xiamen University, Xiamen, Fujian 361005, P. R. China

IDepartment of Chemistry, Agnes Scott College, 141 E College Avenue, Decatur, Georgia 30030, United States

${ }^{\&}$ Center for Functional Nanomaterials, Brookhaven National Laboratory, Upton, New York 11973, United States

*Corresponding author: dong.qin@mse.gatech.edu

Keywords: seed-mediated growth · surface capping · concave nanocrystal · surface-enhanced Raman spectroscopy $\cdot$ Au-catalyzed reduction and oxidation 


\begin{abstract}
We report a facile synthesis of Ag@Au concave cuboctahedra by titrating aqueous $\mathrm{HAuCl}_{4}$ into a suspension of Ag cuboctahedra in the presence of ascorbic acid (AA), $\mathrm{NaOH}$, and poly(vinyl pyrrolidone) (PVP) at room temperature. Initially, the Au atoms derived from the reduction of $\mathrm{Au}^{3+}$ by AA are conformally deposited on the entire surface of a Ag cuboctahedron. Upon the formation of a complete Au shell, however, the subsequently formed Au atoms are preferentially deposited onto the Au\{100\} facets, resulting in the formation of a Ag@Au cuboctahedron with concave structures at the sites of $\{111\}$ facets. The concave cuboctahedra embrace excellent SERS activity that is more than 70-fold stronger than that of the original Ag cuboctahedra at an excitation wavelength of $785 \mathrm{~nm}$. The concave cuboctahedra also exhibit remarkable stability in the presence of an oxidant such as $\mathrm{H}_{2} \mathrm{O}_{2}$ because of the protection by a complete Au shell. These two unique attributes enable in-situ SERS monitoring of the reduction of 4-nitrothiophenol (4NTP) to 4-aminothiophenol (4-ATP) by $\mathrm{NaBH}_{4}$ through a 4,4'-dimercaptoazobenzene (transDMAB) intermediate and the subsequent oxidation of 4-ATP back to trans-DMAB upon the introduction of $\mathrm{H}_{2} \mathrm{O}_{2}$.
\end{abstract}


Noble-metal nanocrystals with concave surfaces embrace unique properties for applications in catalysis, ${ }^{1-3}$ plasmonics, ${ }^{4-5}$ and surface-enhanced Raman spectroscopy (SERS) ${ }^{6,7}$ due to the involvement of high-index facets and sharp corners/edges. For concave nanocrystals made of $\mathrm{Au}$, $\mathrm{Pd}$, and Pt, for example, they have been demonstrated with significantly enhanced catalytic activity on their high-index facets toward a number of reactions owing to the presence of atomic steps and kinks with low coordination numbers in high densities. ${ }^{8-11}$ For Ag or Au concave nanocrystals, they have been reported with considerably improved SERS activity because of the hot spots at their sharpened corners/edges. ${ }^{12,13}$ Although it is straightforward to synthesize $\mathrm{Au@Ag} \mathrm{concave} \mathrm{nanocrystals} \mathrm{via} \mathrm{seed-mediated} \mathrm{growth,}{ }^{14-16}$ it is far more challenging to generate Ag@Au concave nanocrystals because of the inherent galvanic replacement reaction between elemental $\mathrm{Ag}$ and a salt precursor to $\mathrm{Au} .{ }^{17}$

In recent years, a number of groups have successfully demonstrated the deposition of Au on Ag nanocrystals by defeating the galvanic replacement reaction. One strategy involves the retardation of the galvanic replacement reaction. Specifically, Yin et al. used I' ions or sulfite to substantially reduce the reduction potential of $\mathrm{Au}^{3+}$ and thus inhibit the galvanic replacement for the generation of Au-coated Ag nanoplates. ${ }^{18,19}$ Kitaev et al. noted that the galvanic replacement reaction between Ag decahedra or pentagonal nanorods and $\mathrm{HAuCl}_{4}$ could be retarded when they titrated the $\mathrm{HAuCl}_{4}$ solution at an extremely slow rate to minimize the local concentration of $\mathrm{Au}^{3+}$ precursor. ${ }^{20}$ Most recently, we demonstrated that the co-introduction of $\mathrm{Ag}^{+}$ions with $\mathrm{Au}^{3+}$ ions could effectively push the galvanic replacement reaction between $\mathrm{Ag}$ nanocubes and $\mathrm{Au}^{3+}$ backward and thus impede it, achieving the co-deposition of Ag and Au atoms on Ag nanocubes in a fashion essentially identical to seed-mediated growth. ${ }^{21}$

An alternative strategy relies on the introduction of a reducing agent such as ascorbic acid (AA) or $\mathrm{NaBH}_{4}$ to compete with and thus defeat the galvanic replacement reaction. To this end, Mirkin and Xue reported the use of AA to assist the formation of Ag-Au alloy shells on the surfaces of Ag nanoplates. ${ }^{22,23}$ Recently, we successfully extended this concept to the deposition of Au on Ag nanocubes for the generation of Ag@Au core-shell nanocubes. ${ }^{24}$ We demonstrated that the galvanic replacement could be completely blocked when the reduction of $\mathrm{Au}^{3+}$ was dominated by $\mathrm{AA}$. As a result, the $\mathrm{Au}$ atoms derived from the reduction of $\mathrm{Au}^{3+}$ by $\mathrm{AA}$ were deposited on the entire surface of Ag nanocubes, leading to the formation of a conformal, complete Au shell that could protect the Ag core from oxidation by $\mathrm{H}_{2} \mathrm{O}_{2}$. We could adjust the 
thickness of the Au shell by controlling the amount of $\mathrm{HAuCl}_{4}$ relative to the number of $\mathrm{Ag}$ nanocubes involved in the reaction. Because each Ag nanocube was mostly covered by the $\{100\}$ facets, we observed the formation of conventional core-shell nanocrystals without any unusual shape or morphology.

In this work, we demonstrate the synthesis of Ag@Au concave cuboctahedra by depositing $\mathrm{Au}$ on Ag cuboctahedra that are covered by a mix of $\{111\}$ and $\{100\}$ facets at a ratio of 1:1.7 in terms of surface area. In a typical synthesis, we titrate aqueous $\mathrm{HAuCl}_{4}$ into an aqueous suspension containing Ag cuboctahedra, $\mathrm{AA}, \mathrm{NaOH}$, and poly(vinyl pyrrolidone) (PVP). As illustrated in Figure 1, the deposition of Au follows a growth pattern completely different from what we have observed in the case of Ag nanocubes due to the presence of $\{111\}$ facets on the surface of a cuboctahedron. In the very early stage, the reduction of $\mathrm{Au}^{3+}$ by $\mathrm{AA}$ generates $\mathrm{Au}$ atoms, followed by their deposition onto the entire surface of a Ag cuboctahedron because PVP lacks the selectivity in capping different types of Ag surfaces in an aqueous medium. ${ }^{25}$ As a result, the Ag cuboctahedron is transformed into a Ag@Au cuboctahedron. As soon as a complete Au shell is created on the Ag cuboctahedron, however, the subsequently formed $\mathrm{Au}$ atoms will be preferentially deposited onto the $\mathrm{Au}\{100\}$ facets due to the selective passivation of $\mathrm{Au}\{111\}$ facets by PVP in an aqueous solution, ${ }^{26}$ slowing down deposition at the sites of $\{111\}$ facets and eventually leading to the formation of a Ag@Au concave cuboctahedron.

We also demonstrate the concave cuboctahedra as an excellent SERS probe, with an activity more than 70-fold stronger than that of the original Ag cuboctahedra at an excitation wavelength of $785 \mathrm{~nm}$. The concave cuboctahedra also exhibit remarkable stability against oxidative etching by $\mathrm{H}_{2} \mathrm{O}_{2}$ due to the presence of complete Au shells for protection. By combining their SERS activity and chemical stability, we further establish the use of the concave cuboctahedra for insitu SERS monitoring of two Au-catalyzed reactions, i.e., the reduction of 4-nitrothiophenol (4NTP) to 4-aminothiophenol (4-ATP) by $\mathrm{NaBH}_{4}$ through a 4,4'-dimercaptoazobenzne (transDMAB) intermediate and the oxidation of the resultant 4-ATP back to trans-DMAB upon the addition of $\mathrm{H}_{2} \mathrm{O}_{2}$, respectively. Our SERS results confirm that the Au-catalyzed reduction of 4NTP to 4-TP proceeds via a condensation route that involves the formation of trans-DMAB molecules. During adsorption, the 4-NTP molecules are likely oriented parallel to the surface of the nanocrystal, facilitating the formation of trans-DMAB through intra-particle $\mathrm{N}=\mathrm{N}$ bonds on a single concave cuboctahedron. As for the Au-catalyzed oxidation of 4-ATP derived from the 
reduction of 4-NTP, our results indicate that 4-ATP can be oxidized to produce trans-DMAB but not 4-NTP. Interestingly, we also observe some major differences in the SERS spectra of the trans-DMAB derived from the oxidation of 4-ATP and the reduction of 4-NTP, respectively. Our data suggest that the oxidation reaction may involve two concave cuboctahedra, leading to the formation of trans-DMAB molecules via inter-particle $\mathrm{N}=\mathrm{N}$ bonds.

\section{RESULTS AND DISCUSSION}

In the first step, we used Ag cubes of $40.5 \pm 4.4 \mathrm{~nm}$ in edge length (Figure S1A) to generate Ag cuboctahedra with an average size of $48.2 \pm 3.2 \mathrm{~nm}$ (Figure S1B, together with the definition of size) by following a protocol reported in literature. ${ }^{27}$ Different from a nanocube whose surface is mainly covered by $\{100\}$ facets, the surface of a cuboctahedron is enclosed by a mix of $\{111\}$ and $\{100\}$ facets with a ratio of 1:1.7 between their areas. We then dispersed the as-prepared Ag cuboctahedra in an aqueous solution ( $\mathrm{pH}=11$ ) containing $\mathrm{AA}, \mathrm{NaOH}$, and $\mathrm{PVP}$, followed by the titration of aqueous $\mathrm{HAuCl}_{4}$ using a syringe pump at a relatively slow injection rate of 0.02 $\mathrm{mL} / \mathrm{min}$ under the ambient condition. Samples were collected from the system after different volumes of the $\mathrm{HAuCl}_{4}$ solution had been introduced. To elucidate the deposition pattern of $\mathrm{Au}$ atoms on the Ag cuboctahedra, we etched the samples with aqueous $\mathrm{H}_{2} \mathrm{O}_{2}$ to selectively remove the Ag, but not $\mathrm{Au}$, from the nanostructures.

Figure 2 shows SEM and TEM images of the products before and after etching with aqueous $\mathrm{H}_{2} \mathrm{O}_{2}$. After the addition of $0.2 \mathrm{~mL} \mathrm{HAuCl}_{4}$ solution, we observed very little change to the Ag cuboctahedra (Figure 2A). The absence of pits on the surfaces of the cuboctahedra indicates that no galvanic replacement was involved during the introduction of $\mathrm{HAuCl}_{4}$ under the experimental conditions. After $\mathrm{H}_{2} \mathrm{O}_{2}$ etching, we found that the structure and shape of some cuboctahedra were preserved while the others were transformed into nanocages with hollow interiors and porous walls (Figure 2B). In this case, the number of $\mathrm{Au}$ atoms derived from the reduction of $\mathrm{Au}^{3+}$ by AA was insufficient to form a complete shell on every Ag cuboctahedron. Those nanoparticles without complete Au shells evolved into nanocages when the Ag was etched away by $\mathrm{H}_{2} \mathrm{O}_{2}$. By comparing the UV-vis spectra recorded from the samples before and after $\mathrm{H}_{2} \mathrm{O}_{2}$ etching (Figure S2A), we noticed that there was very little change to the localized surface plasmon resonance (LSPR) peak in terms of position while the intensity dropped by almost 60\%. This observation is consistent with the TEM image shown in Figure 2B because the LSPR peak of the nanocages 
should be located in the near-infrared region beyond $800 \mathrm{~nm}$ due to their extremely thin walls, out of the detection range of the spectrometer. ${ }^{28}$

When the volume of added $\mathrm{HAuCl}_{4}$ solution was increased to $0.4 \mathrm{~mL}$, the shape of the $\mathrm{Ag}$ cuboctahedra was largely retained, with no noticeable pits on the surface of each cuboctahedron (Figure 2C). After $\mathrm{H}_{2} \mathrm{O}_{2}$ etching, the concaveness at the sites of $\{111\}$ facets was somewhat increased (Figure 2D). In this case, UV-vis spectra recorded from the sample before and after $\mathrm{H}_{2} \mathrm{O}_{2}$ etching remained essentially the same, with a slight red shift from 454 to $465 \mathrm{~nm}$ for the LSPR peak (Figure S2B). This observation indicates the formation of a complete shell of Au on the surface of each Ag cuboctahedron for the creation of a Ag@Au core-shell cuboctahedron. Since the concaveness at the sites of $\{111\}$ facets just started to appear, we call such a sample “Ag@Au cuboctahedra” in our discussion.

When the titration volume of $\mathrm{HAuCl}_{4}$ solution was further increased to $0.8 \mathrm{~mL}$, the deposition of $\mathrm{Au}$ on the $\{111\}$ facets became less pronounced relative to the $\{100\}$ facets, leading to the formation of Ag@Au cuboctahedra with obvious concaveness at the sites of $\{111\}$ facets (Figure 2E, in particular, the magnified images in the insets). Remarkably, these concave cuboctahedra could survive in an aqueous $\mathrm{H}_{2} \mathrm{O}_{2}$ solution for a period up to $1 \mathrm{~h}$, without showing any changes to their shape or morphology (Figure 2F). This result also indicates the complete protection of the Ag cores by Au shells. The UV-vis spectra shown in Figure S2C further support the excellent stability of these nanostructures against $\mathrm{H}_{2} \mathrm{O}_{2}$ etching. In the following discussion, we refer to such a sample as “Ag@Au concave cuboctahedra”.

We then used aberration-corrected high-angle annular dark-field scanning TEM (HAADFSTEM) to characterize the Ag@Au concave cuboctahedra. Figure 3A shows a HAADF-STEM image taken from an individual concave cuboctahedron that was oriented along the $<110>$ zone axis. Because the contrast is proportional to the atomic number, we were able to resolve the distributions of Au and Ag atoms on both the $\{111\}$ and $\{100\}$ facets, as shown in Figure 3, BD, respectively. Specifically, the projected thicknesses of Au overlayers on the $\{100\}$ and $\{111\}$ facets were 9-10 atomic layers (or $\sim 2 \mathrm{~nm}$ ) and 4-5 atomic layers (or $\sim 1 \mathrm{~nm}$ ), respectively. These results indicate that more $\mathrm{Au}$ atoms could be deposited on the $\{100\}$ facets than the $\{111\}$ facets. Figure 3E shows a HAADF-STEM image of another concave cuboctahedron that was oriented align with the $<100>$ zone axis, clearly showing the Au shell around the Ag core. To resolve the distributions of Ag and Au elements, we conducted STEM-electron energy-loss spectroscopy 
(EELS) mapping with the $\mathrm{M}_{4,5}$ edges of $\mathrm{Ag}$ and $\mathrm{Au}$, respectively. Figure $3 \mathrm{~F}$ shows the distributions of $\mathrm{Ag}$ and $\mathrm{Au}$ for the concave cuboctahedron shown in Figure 3E, confirming the uniform deposition of $\mathrm{Au}$ on the $\{100\}$ facets. Figure S4 shows the individual EELS mapping with the $\mathrm{M}_{4,5}$ edges of $\mathrm{Ag}$ and Au, respectively. Collectively, the HAADF-STEM and STEMEELS results strongly support our claim that more $\mathrm{Au}$ atoms were deposited on the $\{100\}$ facets for the formation of Ag@Au cuboctahedra with a concaved structure on the site of $\{111\}$ facet. It should be pointed out that the resistance of our Ag@Au cuboctahedra (without or with concaved surfaces) against $\mathrm{H}_{2} \mathrm{O}_{2}$ etching can be attributed to the formation of a complete Au shell around each cuboctahedron. During the deposition of $\mathrm{Au}$, some Ag atoms could be mixed into the $\mathrm{Au}$ shell through inter-diffusion and/or other mechanisms. ${ }^{24,29}$ However, the atomic ratio of Ag to Au should be an extremely small number although it is still impossible to determine the exact value by EDX mapping or HAADF-STEM.

We also used inductively-coupled plasma mass spectrometry (ICP-MS) to quantify the Au content in the as-obtained samples after the Ag cuboctahedra had been titrated with different volumes of $\mathrm{HAuCl}_{4}$ solution. Specifically, we collected the solid products via centrifugation, followed by their dissolution in aqua regia for ICP-MS analysis to determine the Au content. The masses of $\mathrm{Au}$ in the solid products increased linearly with the volume of $\mathrm{HAuCl}_{4}$ solution titrated into the suspension of Ag cuboctahedra (Figure S3, solid line). By assuming that the added $\mathrm{Au}^{3+}$ precursor was completely reduced to Au atoms for their deposition onto the Ag cuboctahedra, we calculated the mass of Au at different titration volumes (Figure S3, dotted line). These results indeed support our argument that the added $\mathrm{HAuCl}_{4}$ was completely reduced by AA to generate Au atoms, followed by their deposition onto the Ag cuboctahedra.

By combining the data from SEM, TEM, HAADF-STEM, and ICP-MS, now we can obtain a good understanding of the pathway that led to the formation of Ag@Au concave cuboctahedra. From the ICP-MS data, the Au atoms derived from the complete reduction of $\mathrm{Au}^{3+}$ ions should be able to form shells of 3 and 6 atomic layers thick when the titration volumes of $\mathrm{HAuCl}_{4}$ were 0.4 and $0.8 \mathrm{~mL}$, respectively, if the Au atoms were assumed to be evenly deposited across the entire surface of a Ag cuboctahedron. However, for the sample obtained at a titration volume of $0.8 \mathrm{~mL}$, the projected thickness (from HAADF-STEM) of Au shell on the $\{111\}$ facets were 910 atomic layers, much thicker than the number of 6 derived from the ICP-MS data under the assumption of even deposition, suggesting the formation of a concave structure on the surface. 
From the zoom-in SEM images shown in Figure 2E, it is clear that the $\{100\}$ facets had a flat surface while the $\{111\}$ facets were clearly concaved, with majority of the Au atoms being deposited on the edges. Taken together, we can conclude that the majority of the Au atoms were preferentially and evenly deposited on the $\{100\}$ facets once a complete $\mathrm{Au}$ shell had been formed. In comparison, the deposition of Au atoms on the $\{111\}$ facets were retarded upon the formation of a complete Au shell. This observation suggests that PVP could selectively bind to and thus passivate the $\mathrm{Au}\{111\}$ facets in an aqueous medium, ${ }^{26}$ slowing down the deposition of $\mathrm{Au}$ on these facets and eventually leading to the formation of stellated structures as a result of the continuous deposition and extension at the edges and $\{100\}$ facets, but not the $\{111\}$ facets.

We characterized the LSPR properties of the Ag cuboctahedra, Ag@Au cuboctahedra, and Ag@Au concave cuboctahedra using UV-vis spectroscopy. Figure 4A shows UV-vis spectra of the Ag cuboctahedra before and after they had been titrated with 0.4 and $0.8 \mathrm{~mL}$ of the $\mathrm{HAuCl}_{4}$ solution, respectively, followed by their removal from the reaction solution and re-dispersion in DI water for UV-vis characterization. It is worth mentioning that, after correction for the dilution factor resulting from the different titration volumes of the $\mathrm{HAuCl}_{4}$ solution, the change in peak intensity is directly proportional to the variation in extinction coefficient associated with the nanoparticles. At $0.4 \mathrm{~mL}$, the LSPR peak of the products showed a shift from 434 to $454 \mathrm{~nm}$, together with drop in intensity and broadening in width. This observation is consistent with prior reports on Ag-Au bimetallic nanoparticles. ${ }^{30}$ It has been established that the LSPR peaks of Ag$\mathrm{Au}$ bimetallic nanoparticles are always considerably broader than those of the Ag or $\mathrm{Au}$ counterparts because of the scattering at the Ag-Au interface and the difference in frequency dependence for their dielectric constants. At $0.8 \mathrm{~mL}$, the LSPR peak of the products showed a further shift to $458 \mathrm{~nm}$, together with further decrease in intensity and broadening in width. Such changes to the LSPR features are consistent with the findings reported by other groups during the formation of concave surfaces. ${ }^{12}$

We evaluated the SERS properties of the Ag@Au cuboctahedra and concave cuboctahedra by benchmarking against the Ag cuboctahedra. With excitation at $532 \mathrm{~nm}$, Figure S5 shows the SERS spectra collected from 1,4-benzenedithiol (1,4-BDT) adsorbed on the Ag cuboctahedra before and after they had reacted with 0.4 and $0.8 \mathrm{~mL} \mathrm{HAuCl}_{4}$, respectively. The SERS activity of the Ag cuboctahedra did not change much at all upon the deposition of Au on their surfaces. For example, the intensity of the SERS peak at $1564 \mathrm{~cm}^{-1}$ (the benzene ring mode) was increased 
by $10 \%$ and then decreased by $20 \%$ when the Ag cuboctahedra were switched to Ag@Au cuboctahedra and concave cuboctahedra respectively. This observation is in agreement with prior findings. ${ }^{21,31}$ Typically, the inclusion of Au atoms into Ag nanostructures would result in stronger plasmon damping at visible wavelengths and thus attenuation in SERS intensity because the interband transition edge of Au is located around $500 \mathrm{~nm}$ (or $2.5 \mathrm{eV}$ ). ${ }^{32}$ However, by switching the excitation wavelength from 532 to $785 \mathrm{~nm}$ and thereby eliminating plasma damping, the intensity of the SERS peak at $1564 \mathrm{~cm}^{-1}$ was increased by 15- and 74-fold for the Ag@Au cuboctahedra and concave cuboctahedra related to that of the Ag cuboctahedra (Figure 4B). The corresponding enhancement factors (EFs) associated with the Ag cuboctahedra and Ag@Au concave cuboctahedra were $6.5 \times 10^{4}$ and $4.8 \times 10^{6}$, respectively. In comparison, at excitation of $532 \mathrm{~nm}$, the EFs were only 5.0x10 $10^{4}$ and $4.4 \times 10^{4}$ for the Ag cuboctahedra and Ag@Au concave cuboctahedra, respectively. We believe that the sharpened corners and edges on a concave cuboctahedron could concentrate the electromagnetic field at these sites, leading to enormous SERS signals. ${ }^{12,33}$ A stronger Au-thiolate bond over that of Ag-thiolate could make an additional contribution to the increased SERS signals through a chemical mechanism. ${ }^{34-37}$

By combining the excellent SERS activity of the concave cuboctahedra with the catalytic activity of Au atoms on their surfaces, we further demonstrated a unique SERS probe for in-situ monitoring of the reduction of 4-NTP to 4-ATP by $\mathrm{NaBH}_{4}$ in a colloidal suspension. After the functionalization of the concave cuboctahedra with 4-NTP, we collected a UV-vis spectrum to confirm the preservation of particle morphology and dispersion state (Figure S6). Upon the introduction of $\mathrm{NaBH}_{4}$ to initiate the catalytic reaction, we collected SERS spectra from the same reaction solution at different time points at excitation of $785 \mathrm{~nm}$ and $10 \%$ of the laser output power (Figure 5A). At $t=0$, the SERS spectrum of 4-NTP shows three characteristic vibrational bands (marked by dashed orange lines) at $1108 \mathrm{~cm}^{-1}$ (C-N stretching, $v_{\mathrm{CN}}$ ), $1336 \mathrm{~cm}^{-1}$ (O-N-O stretching, $v_{\mathrm{NO} 2}$ ), and $1572 \mathrm{~cm}^{-1}$ (C-C stretching phenyl-ring, $v_{\mathrm{CC}}$ ), ${ }^{37,38}$ respectively. At $t=6 \mathrm{~min}$, the $v_{\mathrm{NO} 2}$ of 4-NTP was blue-shifted from 1336 to $1330 \mathrm{~cm}^{-1}$, together with slight decrease in intensity. The intensities of $v_{\mathrm{CN}}$ and $v_{\mathrm{CC}}$ decreased while their peak positions were essentially retained. On the other hand, four new bands emerged in the SERS spectrum. Specifically, the band at $1595 \mathrm{~cm}^{-1}$ can be assigned to $v_{\mathrm{CC}}$ of 4-ATP (marked by a dashed black line) and the other

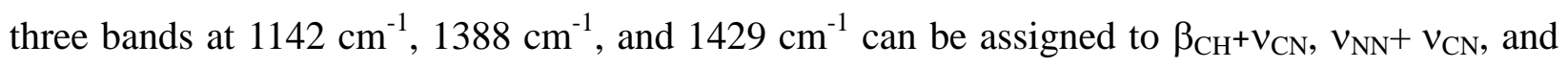


$v_{\mathrm{NN}}+\beta_{\mathrm{CH}}$ of trans-4,4'-dimercaptoazobenzene (trans-DMAB, marked by dashed purple lines), respectively. ${ }^{39,40}$ As the reaction progressed to $14 \mathrm{~min}$ and then $17 \mathrm{~min}$, we noticed that the $v_{\mathrm{NO} 2}$ of 4-NTP (at $1330 \mathrm{~cm}^{-1}$ ) started to decrease in intensity, together with a slight shift to the blue in peak position. Also, the $v_{\mathrm{CC}}$ of 4-NTP (at $1572 \mathrm{~cm}^{-1}$ ) gradually decreased in intensity as that of 4ATP (at $1595 \mathrm{~cm}^{-1}$ ) was continuously increased in intensity. The three bands of trans-DMAB eventually disappeared. At $t=19 \mathrm{~min}$, the bands associated with 4-NTP and trans-DMAB all disappeared. The remaining three peaks can be assigned to the $v_{\mathrm{CS}}, \beta_{\mathrm{C}-\mathrm{H}}$, and $v_{\mathrm{CC}}$ of 4-ATP, respectively. These peaks essentially showed very little change up to $t=45 \mathrm{~min}$. It is also worth noting that the peaks at $1083 \mathrm{~cm}^{-1}$ and $1179 \mathrm{~cm}^{-1}$ can be assigned to 4-NTP, trans-DMAB, or 4ATP, and they were relatively stable in all the SERS spectra during the course of the reaction.

Figure 5B outlines a plausible mechanism for the reduction of 4-NTP to 4-ATP by $\mathrm{NaBH}_{4}$ on a Au surface. Because the SERS spectra indicate that the $v_{\mathrm{CS}}\left(\right.$ at $1083 \mathrm{~cm}^{-1}$ ) of 4-NTP, transDMAB, and 4-ATP were essentially the same during the reduction, we assume that these molecules are preferentially adsorbed at a configuration parallel to the Au surface through the Au-S linkage. ${ }^{41}$ As the reduction by $\mathrm{NaBH}_{4}$ proceeds, the blue-shift for the $v_{\mathrm{NO}_{2}}$ band of 4-NTP indicates a change to the symmetric stretching band of $\mathrm{NO}_{2}$. The assignment of SERS peaks to trans-DMAB rather than cis-DMAB further supports our argument about a parallel configuration for the molecules on a Au surface rather than a vertical configuration. Likely, the first couple of reduction steps would involve the $\mathrm{N}-\mathrm{O}$ bond for the generation of nitro- and hydroxylamine, followed by the condensation of these two intermediates to produce trans-DMAB. ${ }^{42}$ At the end, cleavage of the azo bond due to hydrogenation would lead to the formation of 4-ATP.

By leveraging the remarkable chemical stability of the Ag@Au concave cuboctahedra in 2.3\% aqueous $\mathrm{H}_{2} \mathrm{O}_{2}$, we further used in-situ SERS to investigate the Au-catalyzed oxidation reaction of 4-ATP that was produced by the reduction of 4-NTP on the surface of a concave cuboctahedron. Specifically, we used in-situ SERS to monitor the reduction of 4-NTP by $\mathrm{NaBH}_{4}$ as described previously. Once we observed the SERS peaks of 4-ATP (see Figure 5A at $t=19$ min), we withdrew $300 \mu \mathrm{L}$ of sample from the reaction solution and immediately mixed it with $100 \mu \mathrm{L}$ of $2.3 \%$ aqueous $\mathrm{H}_{2} \mathrm{O}_{2}$. Upon the introduction of $\mathrm{H}_{2} \mathrm{O}_{2}$, we observed a significant decrease in SERS intensity for the reaction mixture. As such, we had to increase the laser power from 10\% (used to collect the SERS spectra shown in Figure 5A) to $100 \%$ of the output in order 
to collect SERS spectra at different time points of the oxidation reaction.

As shown in Figure 6, at $t=2 \mathrm{~min}$, the $v_{\mathrm{CC}}$ and $v_{\mathrm{CS}}$ of 4-ATP were blue-shifted from 1595 and 1083 to 1570 and $1075 \mathrm{~cm}^{-1}$, respectively. Additionally, the intensities of these bands significantly dropped. For example, the intensity of the $v_{C S}$ band was only 200 counts per second (CPS) at a power of $100 \%$ of the laser output. In comparison, during the reduction of 4 NTP to 4-ATP by $\mathrm{NaBH}_{4}$, the intensity of the $v_{\mathrm{CS}}$ band could be maintained at a level of 2,0004,000 CPS at a much lower power of $10 \%$ of the laser output (see Figure 5A). These results suggest that the monolayers of the Raman probe molecules formed on the surfaces of Ag@Au concave cuboctahedra were not stable in the presence of $\mathrm{H}_{2} \mathrm{O}_{2}$. In fact, it has been reported that thiolate-based monolayers are susceptible to oxidation. In general, they tended to de desorbed from the surface upon treatment with $1 \% \mathrm{H}_{2} \mathrm{O}_{2} \cdot{ }^{43,44}$ Concurrently, three characteristic bands of trans-DMAB appeared at 1143, 1393, and $1437 \mathrm{~cm}^{-1}$, indicating the conversion of 4-ATP back to trans-DMAB. Afterwards, the SERS spectra remained essentially the same except some minor changes to their peak intensities up to $t=28 \mathrm{~min}$. From the SERS spectra, we did not observe the peaks associated with 4-NTP. These results suggest that the oxidation of 4-ATP by $\mathrm{H}_{2} \mathrm{O}_{2}$ proceeded rapidly to produce trans-DMAB, which could not be further oxidized to 4-NTP, the original reactant. This observation is consistent with the results of other groups. ${ }^{45,46}$

Because the SERS spectra could provide “fingerprints” for identifying the chemical species attached to the surfaces of the concave cuboctahedra, ${ }^{46-48}$ we studied the Raman shifts for transDMAB molecules that were produced either by reduction of 4-NTP or oxidation of 4-ATP. Interestingly, we found that the two bands associated with the N-N stretching of DMAB, i.e., $\left(v_{\mathrm{NN}}+v_{\mathrm{CN}}\right)$ and $\left(v_{\mathrm{NN}}+\beta_{\mathrm{CH}}\right)$, indicated subtle difference in their peak positions while the band of $\beta_{\mathrm{CH}^{+}}+v_{\mathrm{CN}}$ was located at essentially the same position. For example, the SERS peaks of DMAB molecules derived from the reduction of 4-NTP by $\mathrm{NaBH}_{4}$ were located at $1388\left(v_{\mathrm{NN}}+v_{\mathrm{CN}}\right)$, and $1429 \mathrm{~cm}^{-1}\left(v_{\mathrm{NN}}+\beta_{\mathrm{C}-\mathrm{H}}\right)$ (see Figure $\left.5 \mathrm{~A}\right)$. In comparison, when they were formed via the oxidation of 4-ATP by $\mathrm{H}_{2} \mathrm{O}_{2}$, these peaks were red-shifted to 1393 and $1437 \mathrm{~cm}^{-1}$, respectively. The difference in Raman shifts could infer the formation of trans-DMAB molecules via inter-particle $\mathrm{N}=\mathrm{N}$ bonds that are formed between 4-ATP molecules located on two different concave cuboctahedra, which is consistent with a prior observation. ${ }^{49}$ Likely, a trans configuration can be easily achieved for the trans-DMAB formed via inter-particle linkage, shifting the Raman peaks 
to higher wavenumbers (frequencies). We also noticed that, during the reduction, the intensities of Raman bands for trans-DMAB were comparable to that of the signature band of 4-ATP at $1595 \mathrm{~cm}^{-1}\left(v_{\mathrm{CC}}\right)$ but much weaker than the C-S stretching band of 4-ATP at $1083 \mathrm{~cm}^{-1}\left(v_{\mathrm{CS}}\right.$ ) (see Figure 5A). Once the 4-ATP molecules had been oxidized, the bands of trans-DMAB became dominant in the SERS spectrum (see Figure 6). This data also supports our hypothesis that interparticle linkage occurred during the oxidation of 4-ATP to trans-DMAB. We believe that individual trans-DMAB molecules could be sandwiched between two concave cuboctahedra to

generate significantly enhanced SERS signals due to the involvement of a hot spot. ${ }^{50,51}$ In this case, although the molecules trapped in hot spots contributed most to the SERS signals, they were relatively small in terms of population, making it difficult to use SERS to monitor changes to those molecules outside the hot spots.

\section{Conclusions}

In summary, we have demonstrated the synthesis of Ag@Au concave cuboctahedra by simply titrating aqueous $\mathrm{HAuCl}_{4}$ into a suspension of Ag cuboctahedra enclosed by both $\{100\}$ and $\{111\}$ facets in the presence of $\mathrm{AA}, \mathrm{NaOH}$, and PVP. As the titration volume of $\mathrm{HAuCl}_{4}$ solution was increased, we discovered that the Au atoms were initially deposited on the entire surface of each Ag cuboctahedron, followed by switching to preferential deposition on the $\{100\}$ facets only. These results suggest that PVP did not have selectivity in binding to different types of Ag facets in an aqueous medium but it could selectively passivate the $\mathrm{Au}\{111\}$ facets and thus slow down the deposition at these sites. The Ag@Au concave cuboctahedra exhibited excellent SERS activity that was more than 70-fold stronger than that of the original Ag cuboctahedra at an excitation wavelength of $785 \mathrm{~nm}$. Together with remarkable chemical stability in aqueous $\mathrm{H}_{2} \mathrm{O}_{2}$, we demonstrated that they could serve as an excellent SERS probe for in-situ monitoring of Aucatalyzed reactions, including both reduction and oxidation.

\section{EXPERIMENTAL SECTION}

Chemicals and Materials. Silver nitrate $\left(\mathrm{AgNO}_{3}, 99+\%\right)$, silver trifluoroacetate ( $\mathrm{CF}_{3} \mathrm{COOAg}$, 98\%), gold(III) chloride trihydrate $\left(\mathrm{HAuCl}_{4} \cdot 3 \mathrm{H}_{2} \mathrm{O}, 99.9+\%\right)$, poly(vinyl pyrrolidone) with an average molecular weight of 29,000 (PVP-29) or 55,000 (PVP-55), ethylene glycol (EG, J.T. Baker), sodium hydrosulfide hydrate $\left(\mathrm{NaHS} \cdot \mathrm{xH}_{2} \mathrm{O}\right)$, aqueous 
hydrochloric acid ( $\mathrm{HCl}$, 37\%), sodium citrate tribasic dihydrate $\left(\mathrm{HOC}(\mathrm{COONa})\left(\mathrm{CH}_{2} \mathrm{COONa}\right)_{2} \cdot 2 \mathrm{H}_{2} \mathrm{O}, 99 \%\right)$, L-ascorbic acid (AA, 99\%), sodium hydroxide ( $\mathrm{NaOH}, 98+\%)$, and hydrogen peroxide $\left(\mathrm{H}_{2} \mathrm{O}_{2}, 30\right.$ wt.\% in $\left.\mathrm{H}_{2} \mathrm{O}\right)$ were all purchased from SigmaAldrich and used as received. All aqueous solutions were prepared using deionized (DI) water with a resistivity of $18.2 \mathrm{M} \Omega \cdot \mathrm{cm}$.

Synthesis of Ag Nanocubes. The Ag nanocubes were prepared using a polyol method, with EG and $\mathrm{CF}_{3} \mathrm{COOAg}$ serving as a solvent and a precursor to elemental silver, respectively. In a typical synthesis, $5 \mathrm{~mL}$ of EG was introduced into a round bottom flask (100 mL, ACE Glass) and heated under magnetic stirring in an oil bath held at $150{ }^{\circ} \mathrm{C}$, followed by the introduction of $0.06 \mathrm{~mL}$ of NaHS (3 mM in EG). After $2 \mathrm{~min}, 0.5 \mathrm{~mL}$ of $\mathrm{HCl}$ (3 mM in EG) was introduced, followed by the addition of $1.25 \mathrm{~mL}$ of PVP-55 (20 mg/mL in EG). After another $2 \mathrm{~min}, 0.4 \mathrm{~mL}$ of $\mathrm{CF}_{3} \mathrm{COOAg}$ (282 $\mathrm{mM}$ in EG) was injected into the mixture. The flask was capped with a glass stopper, except for the introduction of reagents. We obtained Ag nanocubes of $40.5 \pm 4.4 \mathrm{~nm}$ in edge length by quenching the reaction with an ice-water bath as soon as the major UV-vis absorption peak of the suspension was shifted to $434 \mathrm{~nm}$. After centrifugation and washing with acetone and DI water three times, the Ag nanocubes were dispersed and stored in DI water.

Synthesis of Ag Cuboctahedra. We used the Ag nanocubes with edge length of $40.5 \pm 4.4 \mathrm{~nm}$ as seeds to obtain Ag cuboctahedra. In a typical synthesis, $50 \mu \mathrm{L}$ of the aqueous suspension of Ag nanocubes $\left(6.6 \times 10^{12}\right.$ particles per $\left.\mathrm{mL}\right)$ was introduced into an aqueous solution containing 5 mL PVP-55 (2 mg/mL), $50 \mu \mathrm{L}$ sodium citrate $(20 \mathrm{mM})$, and $100 \mu \mathrm{L}$ aqueous AA (100 mM) hosted in a $20 \mathrm{~mL}$ glass vial. Next, $1.5 \mathrm{~mL}$ of aqueous $\operatorname{AgNO}_{3}(1 \mathrm{mM})$ was titrated into the mixture using a syringe pump at a rate of $5 \mathrm{~mL} / \mathrm{h}$ under magnetic stirring at room temperature. During the reaction, the major LSPR peak of the cubic seeds showed a slight red-shift from 434 to $436 \mathrm{~nm}$ whereas the peak at $380 \mathrm{~nm}$ for the cubic seeds disappeared during the formation to cuboctahedra. Finally, we collected the supernatant by centrifugation at 1,000 rpm to remove the impurity of large particles, followed by the collection of cuboctahedra by centrifugation of the aforementioned supernatant at 8,000 rpm. We washed the particles with DI water three times and then have them re-dispersed in $100 \mu \mathrm{L}$ DI water for future use.

Synthesis of Ag@Au Cuboctahedra and Concave Cuboctahedra. In a standard synthesis, $2 \mathrm{~mL}$ of an aqueous solution of PVP-29 (1 mM, in terms of the polymer) was introduced into a 
$20 \mathrm{~mL}$ glass vial, followed by the addition of $0.5 \mathrm{~mL}$ of aqueous $\mathrm{AA}(100 \mathrm{mM}), 0.5 \mathrm{~mL}$ of aqueous $\mathrm{NaOH}(200 \mathrm{mM})$, and $30 \mu \mathrm{L}$ of a suspension of the as-prepared Ag cuboctahedra (3.35 mg per mL) under magnetic stirring. Next, $0.4 \mathrm{~mL}$ or $0.8 \mathrm{~mL}$ of aqueous $\mathrm{HAuCl}_{4}(0.08 \mathrm{mM})$ was titrated into the mixture using a syringe pump at a rate of $0.02 \mathrm{~mL} / \mathrm{min}$ for the generation of Ag@Au cuboctahedra and concave cuboctahedra, respectively. After completion of titration, the reaction was allowed to proceed for another $10 \mathrm{~min}$ and the final product was collected by centrifugation at 8,000 rpm and washed with DI water twice prior to SEM characterization.

Etching of the Ag@Au Cuboctahedra and Concave Cuboctahedra. We took out $1 \mathrm{~mL}$ of the as-prepared sample, collected the solid product by centrifugation at 8,000 rpm, washed with DI water once, and then added $1 \mathrm{~mL}$ of aqueous $\mathrm{H}_{2} \mathrm{O}_{2}$ (2.3 wt.\%, diluted from the original 30 wt.\% solution) under magnetic stirring. After $1 \mathrm{~h}$, the solid product was collected by centrifugation at $8,000 \mathrm{rpm}$ and washed with DI three times prior to SEM or TEM characterization.

Instrumentation and Characterization. The UV-vis spectra were collected using a Cary 50 spectrometer (Agilent Technologies, Santa Clara, CA). The quantitative measurement of Ag content was performed using an inductively coupled plasma mass spectrometer (ICP-MS, NexION 300Q, PerkinElmer, Waltham, MA). An Eppendorf 5430 centrifuge was used for collecting and washing solid samples, as well as for the preparation of ICP-MS samples. Scanning electron microscopy (SEM) images were captured using a Hitachi-SU 8230 cold field emission microscope (Hitachi, Tokyo, Japan) operated at $20 \mathrm{kV}$. Transmission electron microscopy (TEM) images were taken using a Hitachi HT7700 microscope (Hitachi, Tokyo, Japan) operated at $120 \mathrm{kV}$. The high-resolution HAADF-STEM imaging and STEM-EELS mapping were conducted on a Hitachi HD2700C STEM operated at $200 \mathrm{kV}$ and equipped with a probe aberration corrector. For HAADF-STEM imaging, collection angles from 64-341mrad were used. Ag $\mathrm{M}_{4,5}$ edges at $367 \mathrm{eV}$ and $\mathrm{Au} \mathrm{M}_{4,5}$ edges at $2206 \mathrm{eV}$ were collected using a Gatan Enfina ER spectrometer. For STEM-EELS mapping, we scanned an area of 100x100 pixels with a total collection time of $12 \mathrm{~min}$.

Surface-Enhanced Raman Scattering (SERS) Measurements. The Ag and Ag@Au cuboctahedra were functionalized with a $0.6 \mathrm{mM}$ ethanol solution of 1,4-BDT for $1 \mathrm{~h}$. After washing with DI water twice, the 1,4-BDT-functionalized nanoparticles were re-dispersed in water to attain a concentration of approximately $3.3 \times 10^{10}$ particles $/ \mathrm{mL}$. The Raman spectra were 
recorded using a Renishaw inVia Raman spectrometer coupled with a Leica microscope using a 100x objective. The excitation wavelength was 532 or $785 \mathrm{~nm}$, in conjunction with a grating of 2,400 or 1,200 lines/mm, at a power of $10 \%$ of the laser output (50 or $100 \mathrm{~mW}$ ). Data was collected from the solution phase with a collection time of $30 \mathrm{~s}$ for all samples. A sample cell was fabricated from a polydimethylsiloxane (PDMS) block by punching a small hole to hold 20 $\mu \mathrm{L}$ of liquid sample. After the PDMS was attached to a glass slide, we loaded the sample solution and then placed a glass coverslip of $170 \mu \mathrm{m}$ in thickness on top of the PDMS to prevent solvent evaporation. The cover slip also served as a reference point that allowed us to ensure the focal plane was positioned $200 \mu \mathrm{m}$ into the sample.

We used the SERS peak at $1565 \mathrm{~cm}^{-1}$ (the benzene ring mode, 8a) for the 1,4-BDT molecule to calculate the SERS enhancement factor (EF) using the following equation:

$$
E F=\left(I_{\text {SERS }} \times N_{\text {bulk }}\right) /\left(I_{\text {bulk }} \times N_{\text {SERS }}\right)
$$

where $I_{\text {SERS }}$ and $I_{\text {bulk }}$ are the intensities of the 8a band of 1,4-BDT for the SERS and the ordinary spectra from a bulk sample, respectively. $N_{\text {SERS }}$ and $N_{\text {bulk }}$ are the numbers of probe molecules for a SERS sample and a bulk sample, respectively. $I_{\text {SERS }}$ and $I_{\text {bulk }}$ were determined by the areas of the 8a bands. $N_{\text {bulk }}$ was determined from the ordinary Raman spectrum of a $0.1 \mathrm{M} 1,4-\mathrm{BDT}$ solution in $12 \mathrm{M}$ aqueous $\mathrm{NaOH}$ and the focal volume of the Raman system. For these measurements, we used the same set of parameters to collect the ordinary Raman spectra and the solution-phase SERS spectra. As a result, the focal volume remained the same for all measurements.

\section{In-situ SERS Monitoring of the Reduction of 4-Nitrothiophenol by Ag@Au Concave} Cuboctahedra. The Ag@Au concave cuboctahedra were dispersed in $5 \mathrm{~mL}$ of DI water containing 4-nitrothiophenol (4-NTP, $10^{-6} \mathrm{M}$ ) and incubated at room temperature for $1 \mathrm{~h}$. The NTP-functionalized Ag@Au concave cuboctahedra were then washed with DI water twice and re-suspended in DI water to achieve a concentration of approximately $1.3 \times 10^{11}$ particles $/ \mathrm{mL}$. Upon mixing $200 \mu \mathrm{L}$ of the suspension with $200 \mu \mathrm{L}$ of aqueous $\mathrm{NaBH}_{4}(1 \mathrm{mg} / \mathrm{mL})$ in a $1.5 \mathrm{~mL}$ centrifuge tube, the concave cuboctahedra were allowed to catalyze the NTP reduction reaction at room temperature. We withdrew $15 \mu \mathrm{L}$ of sample from the reaction solution every several minutes and placed this sample in a PDMS-based cell for monitoring the progress of the reaction 
by SERS. Successive SERS spectra were collected until complete reduction of 4-NTP to 4-ATP. All SERS spectra were recorded from the solution phase using a $5 \mathrm{x}$ objective. The data was collected with a collection time of $10 \mathrm{~s}$ at a power of $10 \%$ of the output of $785 \mathrm{~nm}$ laser (100 $\mathrm{mW})$.

\section{In-situ SERS Monitoring of the Oxidation of 4-Aminothiophenol Catalyzed by Ag@Au} Concave Cuboctahedra. When the reduction of 4-NTP to 4-ATP was completed, $300 \mu \mathrm{L}$ of the reaction solution was taken out and mixed with $100 \mu \mathrm{L}$ of $2.3 \%$ aqueous $\mathrm{H}_{2} \mathrm{O}_{2}$. Again, we withdrew $15 \mu \mathrm{L}$ of sample from the reaction solution every several minutes and SERS spectrum was collected from the sample to monitor the oxidation 4-ATP using a 50x objective with a long working distance. All SERS spectra were recorded from the solution phase with laser excitation at $785 \mathrm{~nm}$. The data was collected with a collection time of $90 \mathrm{~s}$ at a power of $100 \%$ of the output of $785 \mathrm{~nm}$ laser $(100 \mathrm{~mW})$.

\section{ASSOCIATED CONTENT}

Supporting Information Available: SEM images; UV-vis spectra; ICP-MS results; and EELS data. This material is available free of charge via the Internet at http://pubs.acs.org.

\section{AUTHOR CONFIRMATION}

Corresponding Author

dong.qin@mse.gatech.edu

Notes

The authors declare no competing financial interest.

\section{ACKNOWLEDGEMENT}

This work was supported in part by the National Science Foundation (CHE-1412006), start-up funds from the Georgia Institute of Technology, and 3M non-tenured faculty award. Part of the research was performed at the Institute of Electronics and Nanotechnology (IEN). We thank Ming Luo for performing the ICP-MS analysis. J. Zhang was also partially supported by the China Scholarship Council. S. Winget was on sabbatical leave from the Department of Chemistry at Agnes Scott College. We acknowledge the use of electron microscopy resources at the Center for Functional Nanomaterials, a U.S. DOE Office of Science Facility, at Brookhaven National Laboratory under Contract No. DE-SC0012704. 


\section{REFERENCES AND NOTES}

1. Zhang, H.; Jin, M.; Xia, Y. Noble-Metal Nanocrystals with Concave Surfaces: Synthesis and Applications. Angew. Chem. Int. Ed. 2012, 51, 7656-7673.

2. Burda, C.; Chen, X.; Narayanan, R.; El-Sayed, M. A. Chemistry and Properties of Nanocrystals of Different Shapes. Chem. Rev. 2005, 105, 1025-1102.

3. Joo, S. H.; Park, J. Y.; Tsung, C. K.; Yamada, Y.; Yang, P.; Somorjai, G. A. Thermally Stable Pt/Mesoporous Silica Core-Shell Nanocatalysts for High-Temperature Reactions. Nat. Mater. 2009, 8, 126-131.

4. Rycenga, M.; Cobley, C. M.; Zeng, J.; Li, W.; Moran, C. H.; Zhang, Q.; Qin, D.; Xia, Y. Controlling the Synthesis and Assembly of Silver Nanostructures for Plasmonic Applications. Chem. Rev. 2011, 111, 3669-3712.

5. Haes, A. J.; Haynes, C. L.; McFarland, A. D.; Schatz, G. C.; Van Duyne, R. P.; Zou, S. Plasmonic Materials for Surface-Enhanced Sensing and Spectroscopy. MRS Bulletin 2005, 30, 368-375.

6. Hong, J. W.; Lee, S. U.; Lee, Y. W.; Han, S. W. Hexoctahedral Au Nanocrystals with HighIndex Facets and Their Optical and Surface-Enhanced Raman Scattering Properties. J. Am. Chem. Soc. 2012, 134, 4565-4568.

7. Zhang, Q.; Zhou, Y.; Villarreal, E.; Lin, Y.; Zou, S.; Wang, H. Faceted Gold Nanorods: Nanocuboids, Convex Nanocuboids, and Concave Nanocuboids. Nano Lett. 2015, 15, 41614169.

8. Xia, B. Y.; Wu, H. B.; Wang, X.; Lou, X. W. Highly Concave Platinum Nanoframes with High-Index Facets and Enhanced Electrocatalytic Properties. Angew. Chem. Int. Ed. 2013, 52, 12337-12340.

9. Huang, X.; Zhao, Z.; Fan, J.; Tan, Y.; Zheng, N. Amine-Assisted Synthesis of Concave Polyhedral Platinum Nanocrystals Having $\{411\}$ High-Index Facets. J. Am. Chem. Soc. 2011, 133, 4718-4721.

10. Yu, T.; Kim do, Y.; Zhang, H.; Xia, Y. Platinum Concave Nanocubes with High-Index Facets and Their Enhanced Activity for Oxygen Reduction Reaction. Angew. Chem. Int. Ed. 2011, 50, 2773-2777.

11. Peng, Z.; Yang, H. Synthesis and Oxygen Reduction Electrocatalytic Property of Pt-on-Pd Bimetallic Heteronanostructures J. Am. Chem. Soc. 2009, 131, 7542-7543. 
12. Xia, X.; Zeng, J.; McDearmon, B.; Zheng, Y.; Li, Q.; Xia, Y. Silver Nanocrystals with Concave Surfaces and Their Optical and Surface-Enhanced Raman Scattering Properties. Angew. Chem. Int. Ed. 2011, 50, 12542-12546.

13. Rycenga, M.; Langille, M. R.; Personick, M. L.; Ozel, T.; Mirkin, C. A. Chemically Isolating Hot Spots on Concave Nanocubes. Nano Lett. 2012, 12, 6218-6222.

14. Ma, Y. Y.; Li, W.; Cho, E. C.; Li, Z. Y.; Yu, T.; Zeng, J.; Xie, Z. X.; Xia, Y. Au@Ag CoreShell Nanocubes with Finely Tuned and Well-Controlled Sizes, Shell Thicknesses, and Optical Properties. ACS Nano, 2010, 4, 6725-6734.

15. Xie, W.; Herrmann, C.; Kompe, K.; Haase, M.; Schlucker, S. J. Synthesis of Bifunctional $\mathrm{Au} / \mathrm{Pt} / \mathrm{Au}$ Core/Shell Nanoraspberries for in Situ SERS Monitoring of Platinum-Catalyzed Reactions. J. Am. Chem. Soc. 2011, 133, 19302-19305.

16. Huang, J.; Zhu, Y.; Lin, M.; Wang, Q.; Zhao, L.; Yang, Y.; Yao, K. X.; Han, Y. Site-Specific Growth of Au-Pd Alloy Horns on Au Nanorods: A Platform for Highly Sensitive Monitoring of Catalytic Reactions by Surface Enhancement Raman Spectroscopy. J. Am. Chem. Soc. 2013, 135, 8552-8561.

17. Xia, X.; Wang, Y.; Ruditskiy, A.; Xia, Y. 25th Anniversary Article: Galvanic Replacement: A Simple and Versatile Route to Hollow Nanostructures with Tunable and Well-Controlled Properties. Adv. Mater. 2013, 25, 6313-6333.

18. Gao, C.; Lu, Z.; Liu, Y.; Zhang, Q.; Chi, M.; Cheng, Q.; Yin, Y. Highly Stable Silver Nanoplates for Surface Plasmon Resonance Biosensing. Angew. Chem. Int. Ed. 2012, 51, 5629-5633.

19. Liu, H.; Liu, T.; Zhang L.; Han, L.; Gao, C.; Yin, Y. Etching-Free Epitaxial Growth of Gold on Silver Nanostructures for High Chemical Stability and Plasmnic Activity. Adv. Funct. Mater. 2005, 25, 5435-5443.

20. Murshid, N.; Gourevich, I.; Coombs, N.; Kitaev, V. Gold Plating of Silver Nanoparticles for Superior Stability and Preserved Plasmonic and Sensing Properties. Chem. Commun. 2013, 49, 11355-11357.

21. Sun, X.; Qin, D. Co-Titration of $\mathrm{AgNO}_{3}$ and $\mathrm{HAuCl}_{4}$ : A New Route to the Synthesis of Ag@Ag-Au Core-Frame Nanocubes with Enhanced Plasmonic and Catalytic Properties. J. Mater. Chem. C, 2015, 3, 11833-11841.

22. Sanedrin, R. G.; Georganopoulou, D. G.; Park, S.; Mirkin, C. A. Seed-Mediated Growth of 
Bimetallic Prisms. Adv. Mater. 2005, 17, 1027-1031;

23. Shahjamali, M. M.; Bosman, M.; Cao, S. W.; Huang, X.; Saadat, S.; Martinsson, E.; Aili, D.; Tay, Y. Y.; Liedberg, B.; Loo, S. C. J.; Zhang, H.; Boey, F.; Xue, C. Gold Coating of Silver Nanoprisms. Adv. Funct. Mater. 2012, 22, 849-854.

24. Yang, Y.; Liu, J.; Fu, Z. W.; Qin, D. Galvanic Replacement-Free Deposition of Au on Ag for Core-Shell Nanocubes with Enhanced Chemical Stability and SERS Activity. J. Am. Chem. Soc. 2014, 136, 8153-8156.

25. Murshid, N.; Kitaev, V. Role of Poly(vinylpyrrolidone) (PVP) and Other Sterically Protecting Polymers in Selective Stabilization of $\{111\}$ and $\{100\}$ Facets in Pentagonally Twinned Silver Nanoparticles. Chem. Commun. 2014, 50, 1247-1249.

26. Kim do, Y.; Li, W.; Ma, Y.; Yu, T.; Li, Z. Y.; Park, O. O.; Xia, Y. Seed-Mediated Synthesis of Gold Octahedra in High Purity and with Well-Controlled Sizes and Optical Properties. Chem. Eur. J. 2011, 17, 4759-4764.

27. Wang, Y.; Wan, D.; Xie, S.; Xia, X.; Huang, C. Z.; Xia, Y. Synthesis of Silver Octahedra with Controlled Sizes and Optical Properties via Seed-Mediated Growth. ACS Nano 2013, 7, 4586-4594.

28. Skrabalak, S. E.; Au, L.; Li, X.; Xia, Y. Facile Synthesis of Ag Nanocubes and Au Nanocages. Nature Protocol, 2007, 2, 2182-2190.

29. Xia, B. Y.; Wu, H. B.; Wang X.; Lou, X. W. One-Pot Synthesis of Cubic $\mathrm{PtCu}_{3}$ Nanocages with Enhanced Electrocatlytic Activity for the Methanol Oxidation Reaction. J. Am. Chem. Soc. 2012, 134, 13934-13937.

30. Wang, X.; Zhang, Z. Y.; Hartland, G. V. Electronic Dephasing in Bimetallic Gold-Silver Nanoparticles Examined by Single Particle Spectroscopy. J. Phys. Chem. B 2005, 109, 20324-20330.

31. Yang, Y.; Zhang, Q.; Fu, Z. W.; Qin, D. Transformation of Ag Nanocubes into Ag-Au Hollow Nanostructures with Enriched Ag Contents to Improve SERS Activity and Chemical Stability. ACS Appl. Mater. Interfaces 2014, 6, 3750-3757.

32. Johnson, P. B.; Christy, R. W. Optical Constants of the Noble Metals. Phys. Rev. B: Solid State 1972, 6, 4370-4379.

33. Mulvihill, M. J.; Ling, X. Y.; Henzie, J.; Yang, P. Anisotropic Etching of Silver Nanoparticles for Plasmonic Structures Capable of Single-Particle SERS. J. Am. Chem. Soc. 2010, 132, 
268-274.

34. Wu, D. Y.; Liu, X. M.; Duan, S.; Xu, X.; Ren, B.; Lin, S. H.; Tian, Z. Q. Chemical Enhancement Effects in SERS Spectra: A Quantum Chemical Study of Pyridine Interacting with Copper, Silver, Gold and Platinum Metals. J. Phys. Chem. C 2008, 112, 4195-4204.

35. Morton, S. M.; Jensen, L. Understanding the Molecule-Surface Chemical Coupling in SERS. J. Am. Chem. Soc. 2009, 131, 4090-4098.

36. Valley, N.; Greeneltch, N.; Van Duyne, R. P.; Schatz, G. C. A Look at the Origin and Magnitude of the Chemical Contribution to the Enhancement Mechanism of SurfaceEnhanced Raman Spectroscopy (SERS): Theory and Experiment. J. Phys. Chem. Lett. 2013, 4, 2599-2604.

37. Zhang, Q. F.; Blom, D. A.; Wang, H. Nanoporosity-Enhanced Catalysis on Subwavelength Au Nanoparticles: a Plasmon-Enhanced Spectroscopic Study. Chem. Mater. 2014, 26,51315142.

38. Zhao, L. B.; Chen, J. L.; Zhang, M.; Wu, D. Y.; Tian, Z. Q. Theoretical Study on Electroreduction of $p$-Nitrothiophenol on Silver and Gold Electrode Surfaces. J. Phys. Chem. C 2015, 119, 4949-4958.

39. Stuart, C. M.; Frontiera, R. R.; Mathies, R. A. Excited-State Structure and Dynamics of cisand trans-Azobenzene from Resonance Raman Intensity Analysis. J. Phys. Chem. A 2007, 111, 12072-12080.

40. Huang, Y. F.; Wu, D. Y.; Zhu, H. P.; Zhao, L. B.; Liu, G. K.; Ren, B.; Tian, Z. Q. SurfaceEnhanced Raman Spectroscopic Study of p-Aminothiophenol. Phys. Chem. Chem. Phys. 2012, 14, 8485-8497.

41. Mahata, A.; Rai, R. K.; Choudhuri, I.; Singh, S. K. Pathak, B. Direct vs. Indirect Pathway for Nitrobenzene Reduction Reaction on a Ni Catalyst Surface: A Density Functional Study. Phys. Chem. Chem. Phys. 2014, 6, 26365-26374.

42. Blaser, H. A Golden Boost to an old Reaction. Science, 2006, 313, 312-313.

43. Crudden, C. M.; Horton, J. H.; Boralidze, I. I.; Zenkina, O. V.; McLean, A. B.; Drevniok, B.; She, Z.; Kraatz, H.; Mosey, N. J.; Seki, T.; Keske, E. C.; Leake, J. D.; Webb, A. R.-W.; Wu, G. Ultra Stable Self-assembled Monolayers on N-heterocyclic Carbenes on Gold. Nature Chemistry 2014, 6, 409-414.

44. Lee, M-T.; Hsueh, C-C.; Freund, M. S; Ferguson, G. S. Air Oxidation of Self-assembled 
Monolayers on Polycrystalline Gold: the Role of the Gold Substrate. Langmuir 1998, 14, 6419-6423.

45. Huang, Y. F.; Zhu, H. P.; Liu, G. K.; Wu, D. Y.; Ren, B.; Tian, Z. Q. When the Signal Is Not From the Original Molecule to Be Detected: Chemical Transformation of paraAminothiophenol on Ag during the SERS Measurement. J. Am. Chem. Soc. 2010, 132, 92449246.

46. Sun, M.; Xu, H. A Novel Application of Plasmonics: Plasmon-Driven Surface-Catalyzed Reactions. Small 2012, 8, 2777-2786.

47. Xie, W.; Walkenfort, B.; Schlucker, S. Label-Free SERS Monitoring of Chemical Reactions Catalyzed by Small Gold Nanoparticles Using 3D Plasmonic Superstructures. J. Am. Chem. Soc. 2013, 135, 1657-1660.

48. Schlucker, S. Surface-Enhanced Raman Spectroscopy: Concepts and Chemical Applications. Angew. Chem. Int. Ed. 2014, 53, 4756-4795.

49. Tang, X.; Cai, W.; Yang, L.; Liu, J. Montioring Plasmon-Driven Surface Catalyzed Reactions in situ using Time-Depdent Surface-Enhanced Raman Spectroscopy on Single Particles of Hierarchical Peony-Like Silver Microflowers. Nanoscale 2014, 6, 8612-8616.

50. Camden, J. P.; Dieringer, J. A.; Wang, Y.; Masiello, D. J.; Marks, L. D.; Schatz, G. C.; Van Duyne, R. P. Probing the Structure of Single-Molecule Surface-Enhanced Raman Scattering Hot Spots. J. Am. Chem. Soc. 2008, 130, 12616-12617.

51. Etchegoin, P. G.; Le Ru, E. C. A Perspective on Single Molecule SERS: Current Status and Future Challenges. Phys. Chem. Chem. Phys. 2008, 10, 6079-6089. 


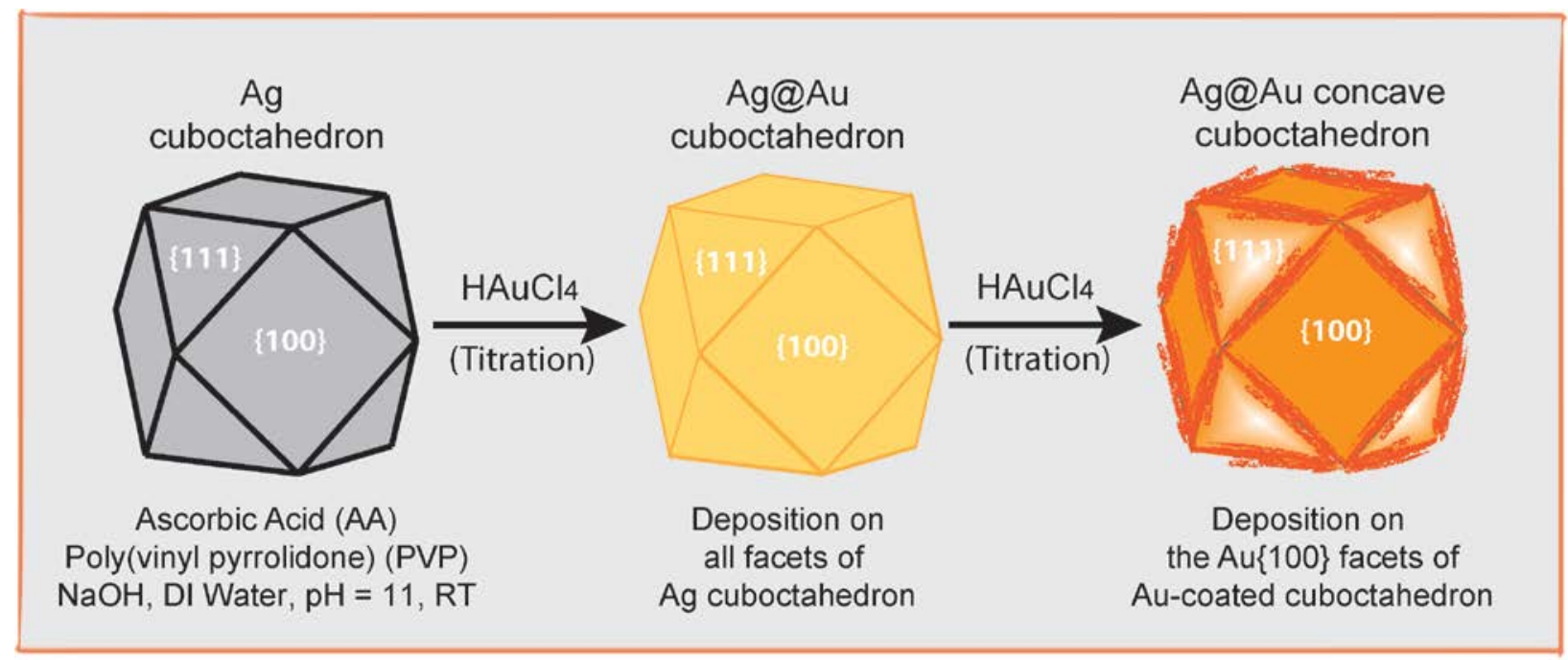

Figure 1. Schematic illustration of a proposed mechanism responsible for the transformation of Ag cuboctahedra into Ag@Au cuboctahedra, and ultimately Ag@Au concave cuboctahedra. 

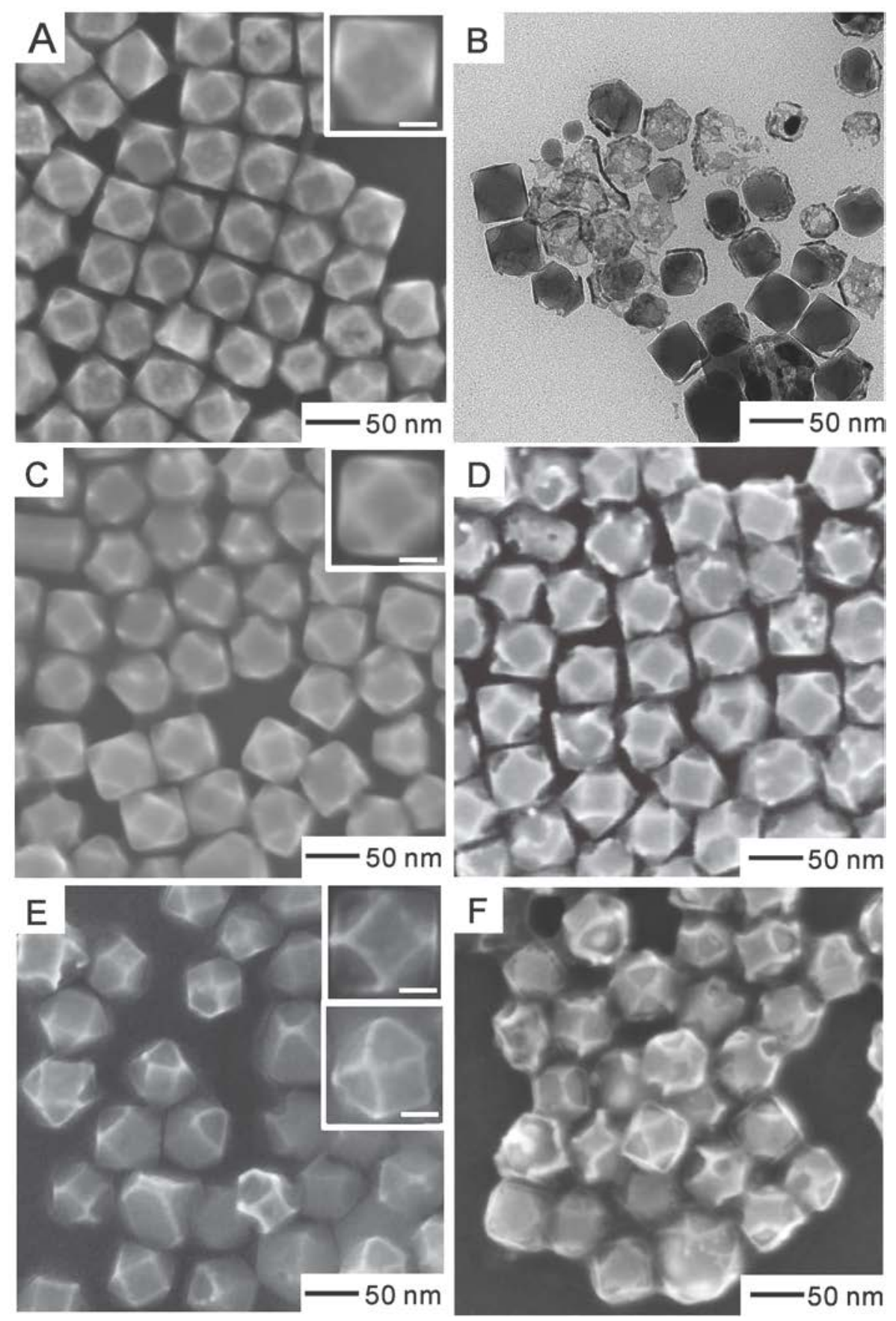

Figure 2. Electron microscopy images of products before (left panel) and after (right panel) etching with 2.3\% aqueous $\mathrm{H}_{2} \mathrm{O}_{2}$. The samples were prepared by reacting Ag cuboctahedra with different volumes of $\mathrm{HAuCl}_{4}$ : (A, B) $0.2 \mathrm{~mL}$, (C, D) $0.4 \mathrm{~mL}$, and (E, F) $0.8 \mathrm{~mL}$, respectively. A TEM image is shown in (B) while the rest is SEM image. Inset scale bar: $20 \mathrm{~nm}$. 

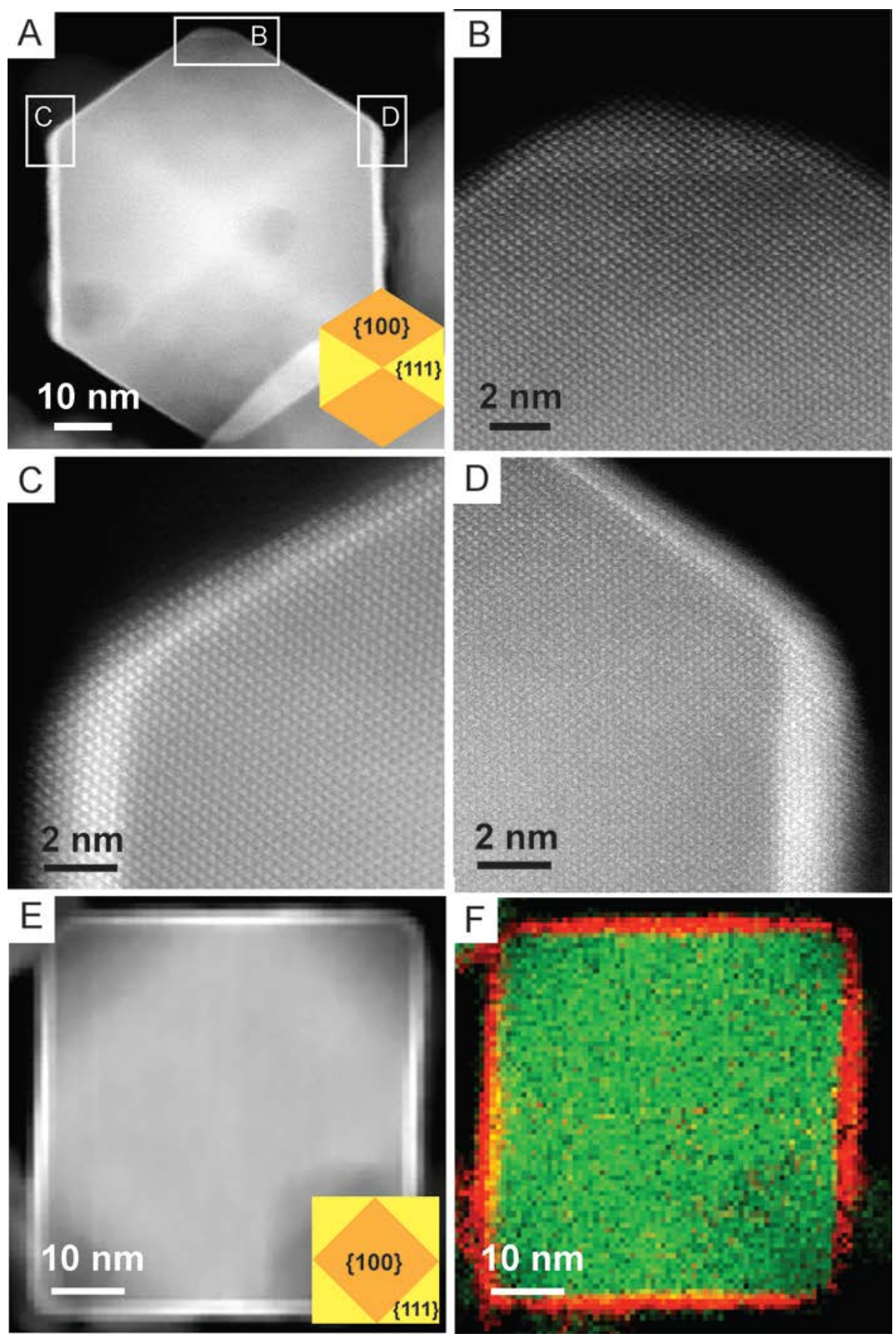

Figure 3. (A-D) HAADF-STEM images taken from one of the Ag@Au concave cuboctahedra shown in Figure 2E when it was oriented along the $<110>$ zone axis. (E) HAADF-STEM image taken from another concave cuboctahedron that was oriented along the $<100>$ zone axis. (F) STEM-EELS mapping of Au (red) and Ag (green) for the cuboctahedron shown in (E). The insets show models of the concave cuboctahedra in the appropriate orientations. 


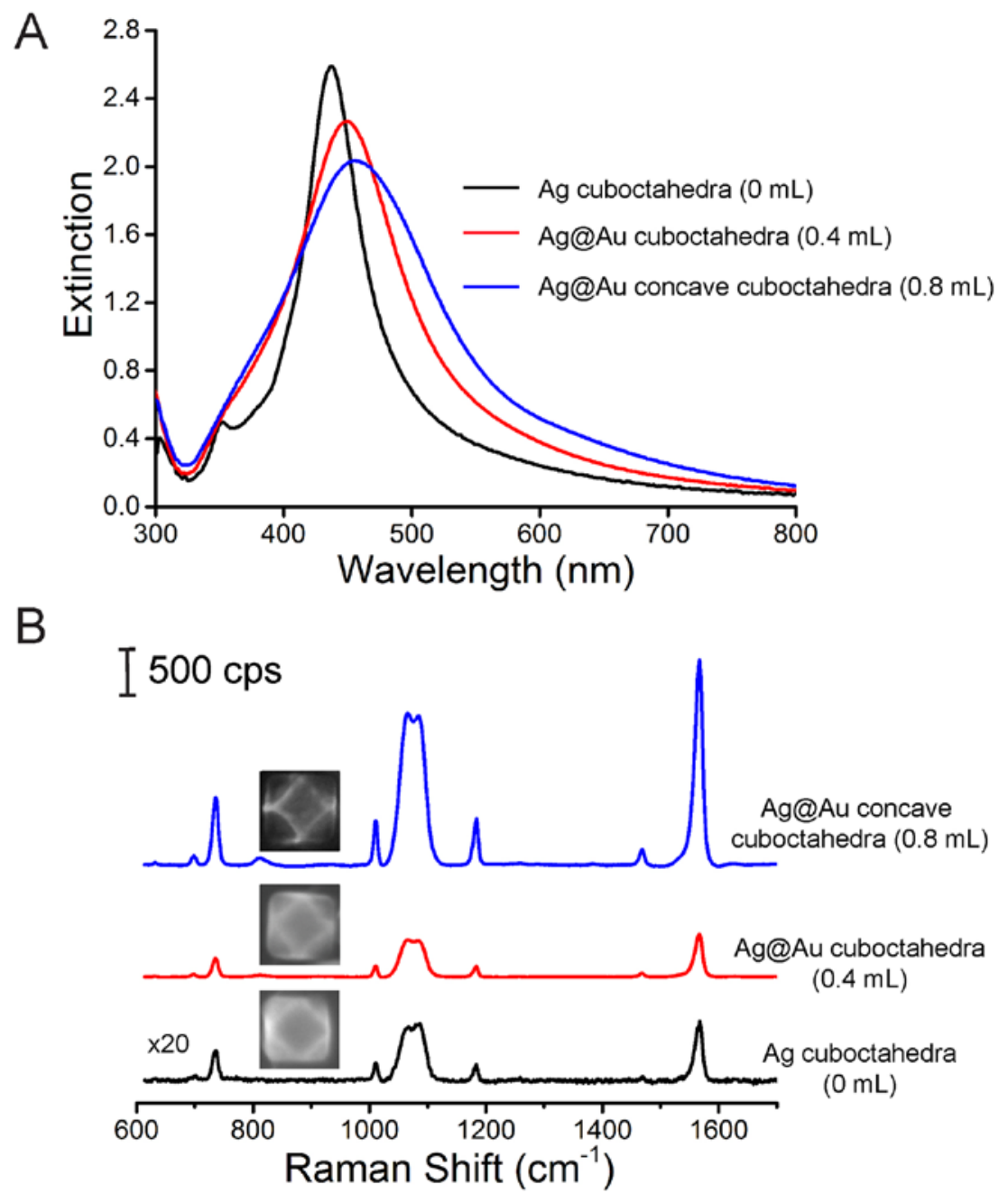

Figure 4. (A) UV-vis spectra of the Ag cuboctahedra, Ag@Au cuboctahedra, and Ag@Au concave cuboctahedra in aqueous suspensions. (B) SERS spectra collected from 1,4-BDT adsorbed on the Ag cuboctahedra, Ag@Au cuboctahedra, and concave cuboctahedra at the excitation of 785 nm. The Ag@Au cuboctahedra and concave cuboctahedra were prepared with the titration of $0.4 \mathrm{~mL}$ and $0.8 \mathrm{~mL}$ aqueous $\mathrm{HAuCl}_{4}$, respectively 


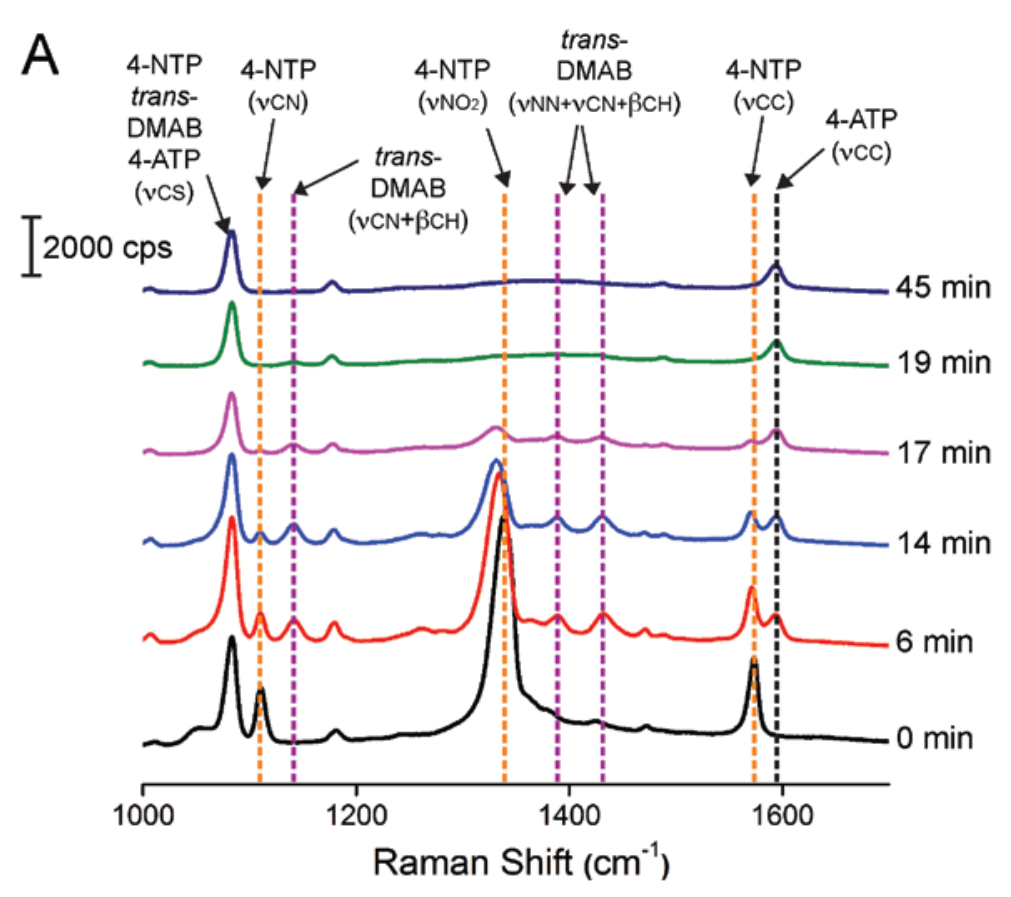

B

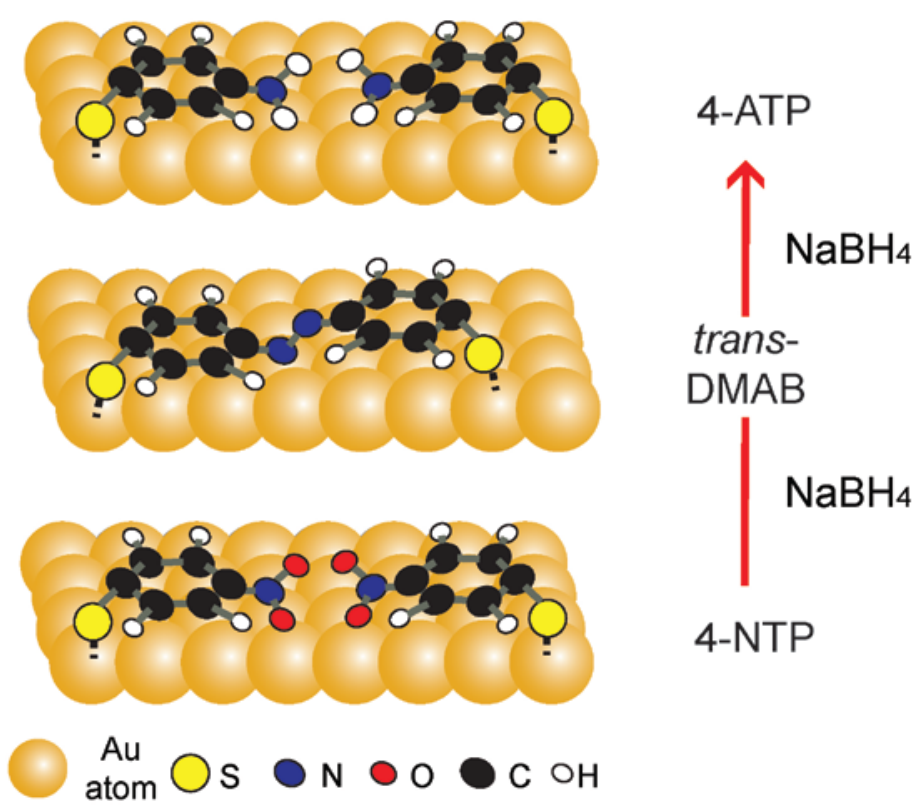

Figure 5. (A) SERS spectra recorded during the reduction of 4-NTP to 4-ATP by $\mathrm{NaBH}_{4}$ at an excitation wavelength of $785 \mathrm{~nm}$. The reaction was catalyzed by the Ag@Au concave cuboctahedra shown in Figure 2E. (B) Schematic illustration (atomic model) of the pathway responsible for the reduction of 4-NTP to 4-ATP on a Au surface. 


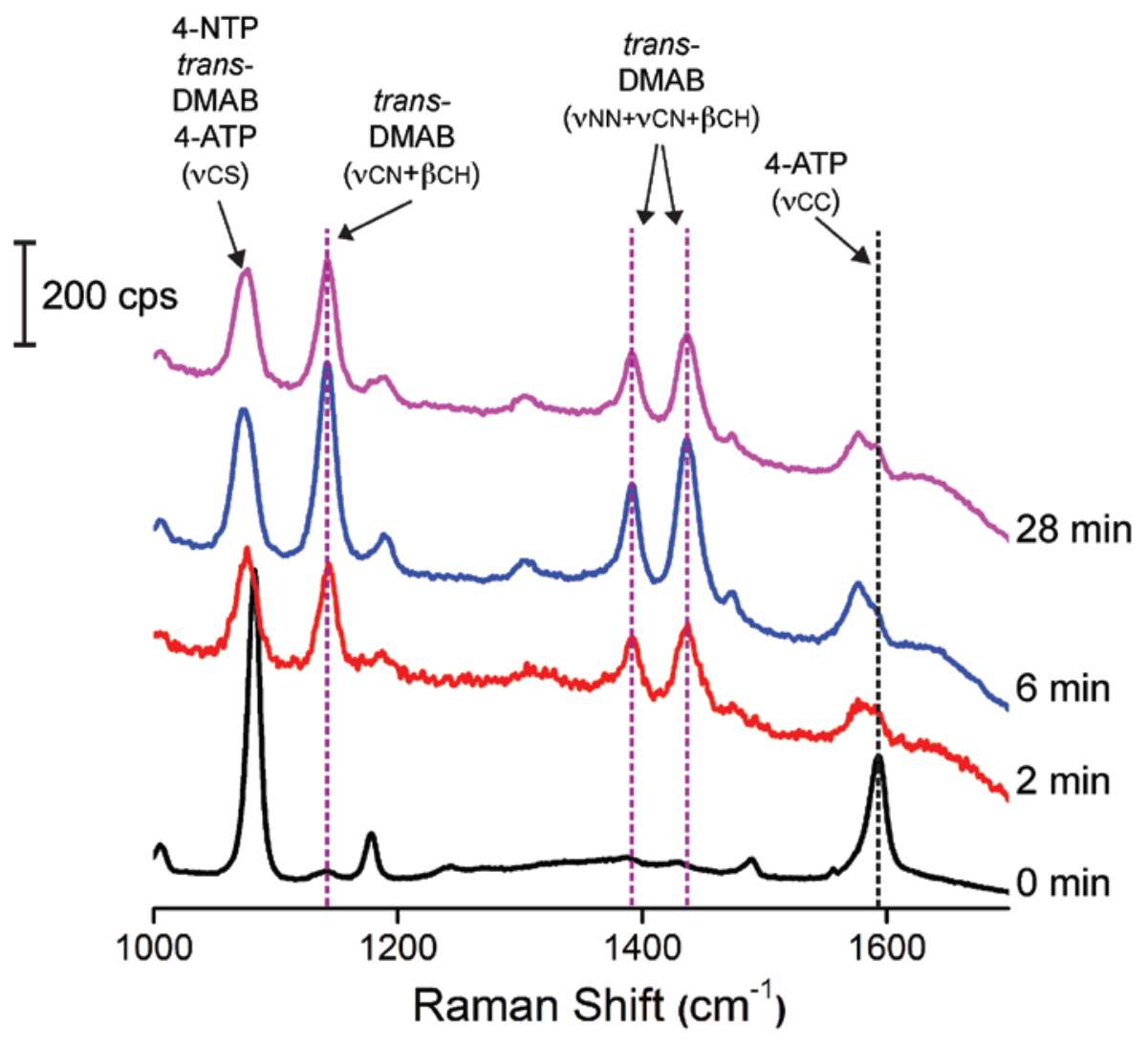

Figure 6. SERS spectra recorded during the oxidation of 4-ATP by $\mathrm{H}_{2} \mathrm{O}_{2}$ at an excitation wavelength of $785 \mathrm{~nm}$. The reaction was catalyzed by the Ag@Au concave cuboctahedra shown in Figure 2E. 


\section{TOC}

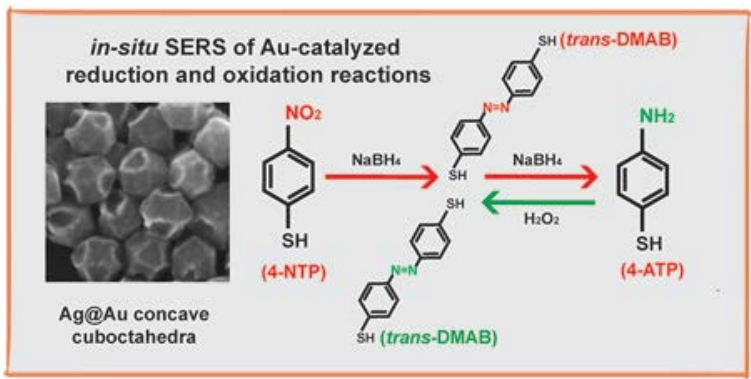

